\title{
Mott Transition of Fermionic Atoms in a Three-Dimensional Optical Trap
}

\author{
R. W. Helmes, ${ }^{1}$ T. A. Costi, ${ }^{2}$ and A. Rosch ${ }^{1}$ \\ ${ }^{1}$ Institute for Theoretical Physics, University of Cologne, 50937 Cologne, Germany \\ ${ }^{2}$ Institute of Solid State Research, Research Centre Jülich, 52425 Jülich, Germany
}

(Received 13 September 2007; published 6 February 2008)

\begin{abstract}
We study theoretically the Mott metal-insulator transition for a system of fermionic atoms confined in a three-dimensional optical lattice and a harmonic trap. We describe an inhomogeneous system of several thousand sites using an adaptation of dynamical mean-field theory solved efficiently with the numerical renormalization group method. Above a critical value of the on-site interaction, a Mott-insulating phase appears in the system. We investigate signatures of the Mott phase in the density profile and in time-offlight experiments.
\end{abstract}

DOI: 10.1103/PhysRevLett.100.056403

PACS numbers: 71.10.Fd, 03.75.Ss, 71.30.th

Introduction. - Ultracold atoms in optical lattices offer exciting possibilities to investigate many-particle effects and to realize and measure models of condensed matter physics like the Hubbard model with unprecedented control of the band structure and interaction strength.

One of the most dramatic effects of strong correlations is the Mott transition, where strong interactions drive a system insulating. While for bosonic atoms [1] the Mott transition in an optical lattice has been realized a few years ago by Greiner et al. [2], the corresponding experiment for fermionic systems turns out to be much more difficult, partially due to problems with cooling and due to the need to work with two hyperfine states, to model the spin degree of freedom. However, due to the enormous experimental progress, a realization of the Mott transition in fermionic systems is expected in the near future. For example, recently Köhl et al. [3] succeeded to capture interacting fermions in an optical lattice and to observe the Fermi surface.

For the interpretation of the experiments, it is essential to investigate the effects of the smooth external confining potential holding the atoms in the trap. The resulting inhomogeneities can make it more difficult to interpret the experiments, but induce also new interesting effects, e.g., associated with the sharp surface between metallic and insulating regions. A frequently used approximation (e.g., in the present context by Ref. [4]) is to describe the trapped atoms locally by a homogeneous system called local density approximation (LDA). However, in the presence of sharp domain walls between two phases, such an approximation is expected to fail and a more realistic treatment of the inhomogeneous system is necessary.

For one-dimensional (1D) systems, powerful numerical [5,6] and analytical [4] methods exists to study theoretically the Mott transition in fermionic systems. For example, in Refs. [4-6], quantum Monte Carlo techniques were used to investigate the signatures of Mott phases in the presence of an external harmonic confinement potential for a 1D system. Rigol et al. [5,6] argued, that in one dimension the inhomogeneities resulting from the trapping potential essentially destroy the main signatures of Mott phases in time-of-flight experiments.

As exact numerical methods for fermions can only be applied to very small systems, one has to resort to approximations to calculate the properties of three-dimensional lattices of realistic size. Here, the method of choice is the so-called dynamical mean-field theory (DMFT) [7,8]. Within DMFT, the only approximation is to neglect nonlocal contributions to the self-energy. This allows us to map the $N$ site lattice problem to $N$ single-impurity Anderson models coupled by a self-consistency condition, see discussion below. DMFT is, for example, frequently used to describe complex bulk materials, e.g., by combining DMFT with band-structure calculations to obtain an $a b$ inito description of strongly correlated materials. In a few cases, DMFT has been employed to describe inhomogeneous systems [9-12] like the surface of Mott insulators [10] or disordered materials [13].

A main problem of DMFT is the need for a reliable and efficient method to solve the effective impurity problems. Previous applications of DMFT to inhomogeneous systems were using impurity solvers like a two-site approximation [10] or slave-boson mean-field theory [13], implying severe further approximations, or started from simplified fermionic models such as the Falicov-Kimball model [12]. We will show that one can also use efficiently one of the most accurate impurity solvers, the numerical renormalization group [14,15] (NRG), to obtain reliable results for traps containing several thousand atoms modeling a fermionic Hubbard model.

After introducing the model and our method (DMFT for inhomogeneous systems + NRG), we will show the resulting spectral functions and discuss how the transition from a metal to a Mott-insulating phase can be seen in real-space and time-of-flight experiments. We investigate the role of temperature, filling, and interaction strength.

Model and Method.-We consider the fermionic Hubbard model on a 3D-cubic lattice, 


$$
\mathcal{H}=-J \sum_{\langle i j\rangle, \sigma} c_{i \sigma}^{\dagger} c_{j \sigma}+U \sum_{i} n_{i \uparrow} n_{i \downarrow}+V_{0} \sum_{i, \sigma} r_{i}^{2} n_{i \sigma}
$$

where $c_{i \sigma}^{\dagger}$ creates a fermion at site $i$ with spin $\sigma, n_{i \sigma}=$ $c_{i \sigma}^{\dagger} c_{i \sigma}$ is the local density, $J$ the nearest-neighbor tunneling matrix element, and $U$ is the effective on-site interaction. The lattice distance, is set to unity. We include 4224 sites with a distance $r_{i} \leq R=10$ from the center of the trap, located in the middle of 8 central sites. The strength of the confining harmonic potential $V_{0}=0.276 \mathrm{~J}$ is chosen such that all sites with $r_{i}>R$ are unoccupied and can be neglected. Using the symmetry of the cubic lattice, one has to deal with only 118 inequivalent sites.

The basic idea of DMFT [7] is to pick a single site of the lattice (the "impurity" $i$ ) and model the effect of all the other sites by a noninteracting bath of fermions. The resulting Anderson impurity model is defined by the local interaction $U$ and the hybridization of the impurity to the bath. The latter is encoded in the $U=0$ Green's function, $G_{\text {And }, i}^{0}(\omega)$. For this model, one determines the local selfenergy $\Sigma_{i}(\omega)$ (see below). From the $\Sigma_{i}(\omega)$, one can construct the lattice Green's function

$$
G_{\mathrm{lat}}^{-1}(\omega)_{i j}=\delta_{i, j}\left[\omega+\mu-\Sigma_{i}(\omega)-V_{0} r_{i}^{2}\right]-J_{i j},
$$

where $J_{i j}=J$ if sites $i$ and $j$ are nearest neighbors and 0 otherwise. The bath of each impurity is then determined from the requirement that at each site the lattice Green's function and the Green's function of the impurity model coincide, $G_{\text {lat }}(\omega)_{i i}=\left[G_{\text {And }, i}^{0}(\omega)^{-1}-\Sigma_{i}(\omega)\right]^{-1}$, thus establishing a self-consistency loop. The scheme described above can be derived using as the only approximation that the self-energy is a local quantity. Both the nonlocal single particle quantum mechanics of fermions and all local effects of strong interactions are correctly described within DMFT.

A main difficulty of DMFT is, however, an accurate calculation of the self-energy of the Anderson impurity model. For this, we use the NRG [16], see Ref. [15] for a description of the method. To obtain efficiently $G_{\text {lat }}$ from an inversion of Eq. (2), it is essential to use the full symmetry of the cubic lattice.

In this Letter, we restrict ourselves to paramagnetic solutions which simplifies the rather challenging numerics considerably. Also experimentally, it is very difficult to reach the low temperatures below which magnetism is expected. Furthermore, we do not expect that magnetic order will change the density profiles or time-of-flight pictures considerably.

Results. - Figure 1 shows how the number of fermions per site evolves for increasing interactions $U / J$, which push the fermions away from the center of the trap. For the chosen parameters, we obtain for $U / D=0,1,2(D=$ $6 J$ is half the bandwidth) a band insulator in the center of the trap and a metal further outside. For the homogeneous system, the critical interaction is given by $U_{c} / D=2.52$,

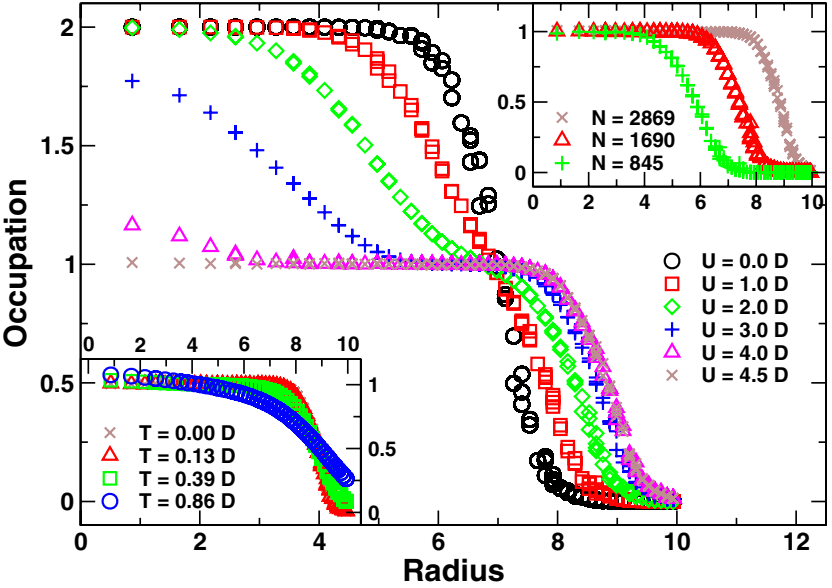

FIG. 1 (color online). The number of fermions $\left\langle n_{i}\right\rangle$ per lattice site, as a function of the distance $r$ to the origin. The signature of the Mott-insulating phase [7] is the presence of a plateau with $\left\langle n_{i}\right\rangle=1$. Note that there are inequivalent sites with different occupation but the same $r$. Main panel: Crossover from weak to strong interactions for a fixed number of $N=2869$ particles in the trap at $T=0, U=6 J$. Upper inset: Dependence on the number of particles for $U=4.5 D, T=0$. Lower inset: $T$ dependence for $U=4.5 D, N=2869$ (the $T=0$ and $T=$ $0.13 D$ curves lie on top of each other).

but already for $U / D=2$, the compressibility close to halffilling is strongly reduced as can be seen in a shoulder in the curve for $\left\langle n_{i}\right\rangle \approx 1$. For $U / D \geq 3$, the incompressibility of the Mott-insulating state, $\partial n / \partial \mu=0$, manifests itself in a plateau. The thickness of this Mott-insulating "onion shell" increases for increasing $U$ eliminating the metallic phase in the center for $U / D=4.5$. The insets of Fig. 1 show how the Mott-insulating region at $U / D=4.5$ shrinks again when the number of fermions in the trap is reduced and how thermal excitations destroy the Mott plateau.

Our method allows us to study the spatial dependence of the spectral functions. While this quantity is difficult to measure for atoms in a trap, it is a highly sensitive probe of the metal-insulator transition. Figure 2 shows how the local spectral function evolves when moving from the center to the edge of the trap at $U / D=4.5$. In the insulating regime, the spectral function $A(\omega)$ is characterized by the two Hubbard bands with equal weight, and $A(\omega=0)$ becomes very small (it never vanishes exactly as atoms from the metallic regions can tunnel into the insulator). As a function of the distance from the center, the Hubbard bands shift due to the harmonic potential. When the Fermi energy starts to merge with one of the Hubbard bands, a sharp quasiparticle peak emerges at $\omega=0$ for sufficiently low $T$ (see inset).

In a time-of-flight experiment, the two-dimensional projection, $n_{\mathbf{k}}^{\text {tof }}=\int n_{\mathbf{k}} d k_{z} /(2 \pi)$, of the three-dimensional momentum distribution 


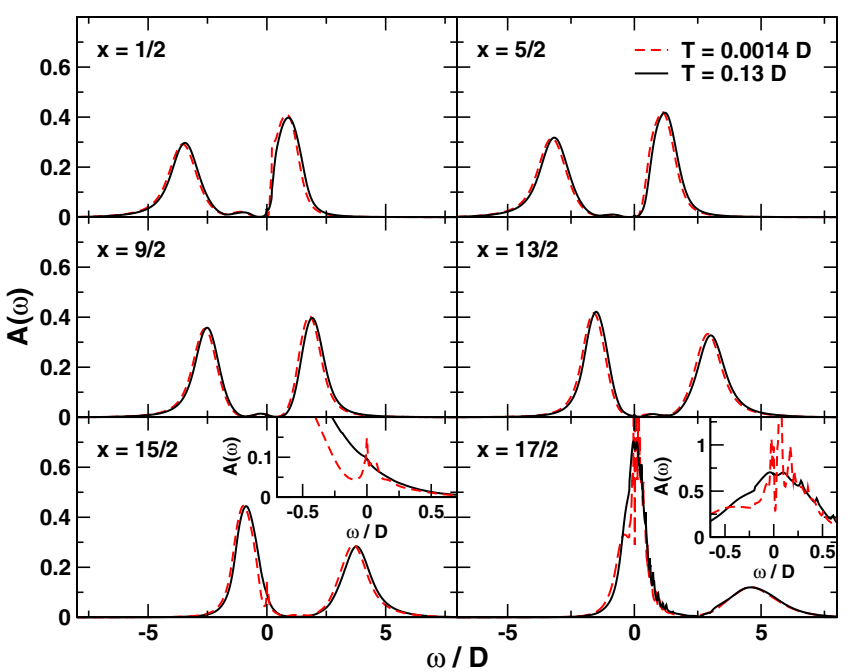

FIG. 2 (color online). Local spectral functions in the Mottinsulating phase $(U=4.5 D, N=2869)$ for two different $T$ for lattice points with coordinates $(x, 1 / 2,1 / 2)$. Left inset: The coherence peak at the Fermi energy, characteristic for a strongly correlated metal, vanishes with increasing $T$. Right inset: Close to edge of the atomic cloud, where the potential becomes steep, (almost) localized states are visible.

$$
n_{\mathbf{k}}=-\frac{1}{N} \sum_{i, j} \int \frac{d \omega}{\pi} f(\omega) e^{i \mathbf{k}\left(\mathbf{r}_{i}-\mathbf{r}_{j}\right)} \operatorname{Im} G_{i j}(\omega)
$$

of the fermions can be measured [2,17]. Here, $f(\omega)$ is the Fermi function, and we have normalized $n_{\mathbf{k}}$ such that $\int n_{\mathbf{k}} \frac{d^{3} \mathbf{k}}{(2 \pi)^{3}}=\int n_{\mathbf{k}}^{\text {tof }} \frac{d^{2} \mathbf{k}}{(2 \pi)^{2}}=1$.

Figure 3 shows $n_{\mathbf{k}}^{\text {tof }}$. For a quantitative analysis of the results and for comparison to (future) experiments, we

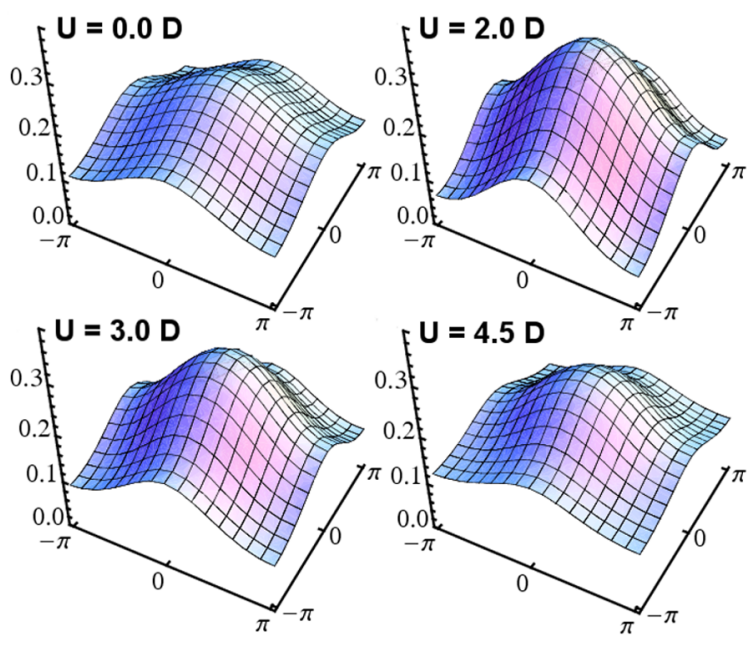

FIG. 3 (color online). Momentum distribution $n_{\mathbf{k}}^{\text {tof }}$ for $U / D=$ $0,2,3,4.5$. Both in the predominantly band-insulating phase $(U / D=0)$ and Mott-insulating phase $(U / D=4.5)$, the curves are considerably flatter than for $U / D=2,3$ where most fermions are in the metallic phase. suggest to plot $n_{\mathbf{k}}^{\text {tof }}$ as a function of the tight-binding dispersion $\epsilon_{\mathbf{k}}^{2 d}=-2 J\left(\cos k_{x}+\cos k_{y}\right)$, see Fig. 4. Surprisingly, each curve collapses to good approximation to a single line, despite the fact that for a given $\epsilon_{\mathbf{k}}^{2 d}$, a range of $n_{\mathbf{k}}^{\text {tof }}$ exists (as is noticeable in the small scatter of the curves for $n_{\mathbf{k}}$ ). Here, it is useful to remember that within DMFT, $n_{\mathbf{k}}$ is only a function of $\epsilon_{\mathbf{k}}$ for the homogeneous system. Therefore, if a LDA were exactly valid, the collapse to a single curve would be perfect. Hence, our results suggest, that LDA is a very good approximation for the analysis of time-of-flight pictures (but not for other quantities, see below).

It is an interesting but difficult question whether this effect is partially an artifact of DMFT which neglects the momentum dependence of the self-energy. While for the experimentally relevant temperature range this is probably a very good approximation, it is expected to fail very close to the metal-insulator transitions at low $T$.

Qualitatively, the results of Fig. 4 reflect that localized electrons in the band- or Mott-insulating phase are characterized by a momentum-independent $n_{\mathbf{k}}$, while in the homogeneous metallic phase, $n_{\mathbf{k}}$ displays a jump at the Fermi momentum with a height given by the quasiparticle weight $Z$. As the effective local Fermi momentum varies smoothly within the trap, all jumps are smeared out.

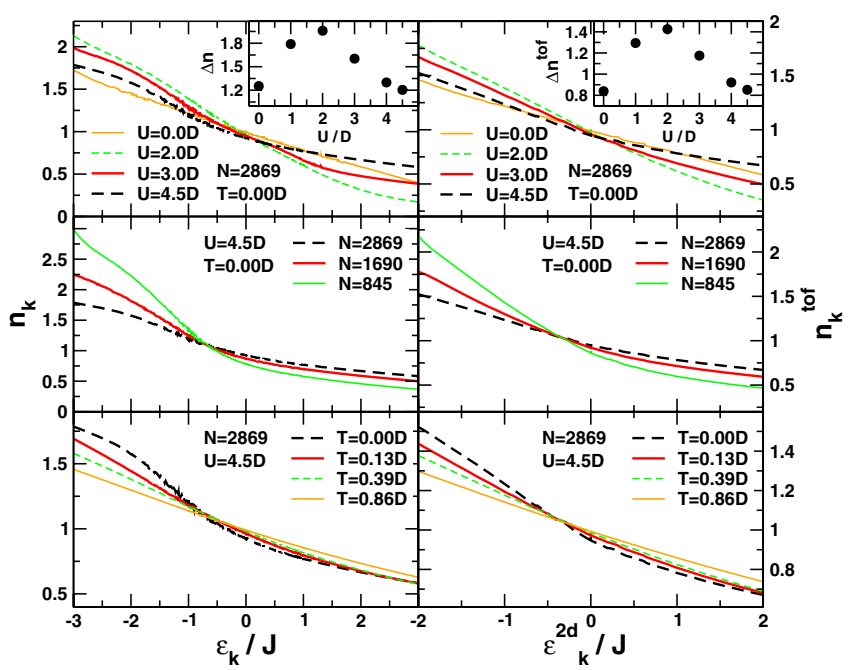

FIG. 4 (color online). $\quad n_{\mathbf{k}}$ (left panel) and $n_{\mathbf{k}}^{\text {tof }}$ (right panel) plotted as a function of $\epsilon_{\mathbf{k}}=-2 J\left(\cos k_{x}+\cos k_{y}+\cos k_{z}\right)$ and $\epsilon_{\mathbf{k}}^{2 d}=-2 J\left(\cos k_{x}+\cos k_{y}\right)$, respectively, for different values of $U, N$, and $T$ (upper, middle, and lower panels, respectively). We have sampled discretized $\mathbf{k}$-values of the entire first Brillouine zone, $\mathbf{k}=\pi / 10\left(n_{x}, n_{y}, n_{z}\right), n_{x, y, z}=-10, \ldots, 10$. For different $\mathbf{k}$ with the same $\epsilon_{\mathbf{k}}$, a range of $n_{\mathbf{k}}$ exists (visible, e.g., in the scatter of the black dashed curves in the left panels). This spread is, however, tiny; to a good approximation, the data collapse to lines. Inset: $\Delta n=n_{0,0,0}-n_{\pi, \pi, \pi}\left(\right.$ and $\left.\Delta n^{\text {tof }}=n_{0,0}^{\text {tof }}-n_{\pi, \pi}^{\text {tof }}\right)$ as a function of $U . \Delta n$ and $\Delta n^{\text {tof }}$ are largest for the predominantly metallic phases and smallest for phases with a large band- (small $U$ ) or Mott-insulating (large $U$ ) regions. 


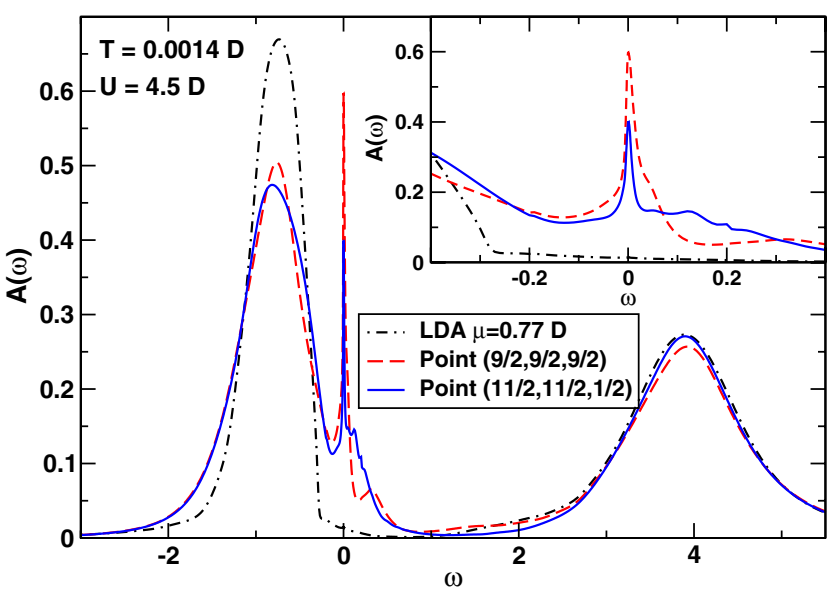

FIG. 5 (color online). Local spectral function of two nonequivalent lattice points at distance 7.794 from the origin at the boundary of the Mott-insulating region $(T=0.0014 D, U=$ $4.5 D$ ). Here, the LDA (dot-dashed line) fails completely to describe the spectral function but still reproduces the occupation with high accuracy $\left(n_{1 / 2(9,9,9)}=0.481, n_{1 / 2(11,11,1)}=0.482\right.$, $\left.n_{\mathrm{LDA}}=0.496\right)$.

However, the slope of $n\left(\epsilon_{\mathbf{k}}\right)$ or the difference $\Delta n^{\text {tof }}=$ $n_{0,0}^{\text {tof }}-n_{\pi, \pi}^{\text {tof }}$ is still a good measure of how metallic or insulating the system is. The inset of Fig. 4 describes the evolution from a mainly band-insulating via a dominately metallic to a Mott-insulating regime when $U / D$ is increased. Similarly, the middle panel of Fig. 4 shows how $\Delta n^{\text {tof }}$ increases when at large $U / D$, the number of particles and therefore the size of the Mott-insulating region is reduced (compare with upper inset of Fig. 1). For increasing temperature (lower panel of Fig. 4), the destruction of quantum coherence leads to a flattening of $n_{\mathbf{k}}^{\text {tof }}$. Note that $n_{\mathbf{k}}^{\text {tof }}$ is more sensitive to changes of $T$ compared to $\left\langle n_{i}\right\rangle$, see Fig. 1.

Conclusions. - In this Letter, we investigated the signatures of the Mott transition of fermions in an optical trap using a local approximation to the self-energy (spaceresolved DMFT + NRG) which allows us to treat several thousand atoms. The clearest signature of a Mott phase is a plateau in the density profile $n(\mathbf{r})$ of the atoms, see Fig. 1 . These plateaus are, however, washed out if only the column density, $\int d z n(\mathbf{r})$, which can be measured directly, is considered (not shown). The Mott transition is more difficult to observe in a time-of-flight experiment. However, the insets of Fig. 4 show that a characteristic flattening of $n_{\mathbf{k}}^{\text {tof }}$ can be seen when a large fraction of the trap becomes a band or Mott insulator.

Our calculations did not rely on a LDA which allows us to investigate whether this widely used approximation is valid in the present context. It turns out that both density profiles and TOF experiments are rather well described by LDA. As discussed in the introduction, LDA is expected not to be valid close to a sharp domain boundary. Indeed, Fig. 5 shows that the LDA fails completely to describe the low-energy excitation spectrum at the boundary of the Mott-insulating region. The coherence peak at the Fermi energy arises due to the penetration of the metallic phase into the Mott insulator via the Kondo effect.

For the future, it will be interesting to investigate the effects of magnetism. In systems with a population imbalance, we expect that the majority spin will accumulate in the Mott-insulating regions. These effects will be studied in a forthcoming publication.

We acknowledge useful discussions with L. Craco, T. Micklitz, D. van Oosten, H. Tjeng, M. Vojta, supercomputer support by the John von Neumann institute for Computing (Jülich) and the Regional Computing Center Cologne and financial support by the SFB 608 of the DFG.

[1] D. Jaksch et al., Phys. Rev. Lett. 81, 3108 (1998).

[2] M. Greiner et al., Nature (London) 415, 39 (2002).

[3] M. Köhl et al., Phys. Rev. Lett. 94, 080403 (2005).

[4] X.-J. Liu, P.D. Drummond, and H. Hu, Phys. Rev. Lett. 94, 136406 (2005).

[5] M. Rigol, A. Muramatsu, G. G. Batrouni, and R.T. Scalettar, Phys. Rev. Lett. 91, 130403 (2003).

[6] M. Rigol, R. T. Scalettar, P. Sengupta, and G. G. Batrouni, Phys. Rev. B 73, 121103 (2006).

[7] A. Georges, G. Kotliar, W. Krauth, and M. J. Rozenberg, Rev. Mod. Phys. 68, 13 (1996).

[8] W. Metzner and D. Vollhardt, Phys. Rev. Lett. 62, 324 (1989).

[9] V. Dobrosavljević and G. Kotliar, Phys. Rev. Lett. 78, 3943 (1997).

[10] S. Okamoto and A. J. Millis, Nature (London) 428, 630 (2004); Phys. Rev. B 70, 241104 (2004).

[11] M. Potthoff and W. Nolting, Phys. Rev. B 59, 2549 (1999); S. Schwieger, M. Potthoff, and W. Nolting, ibid. 67, 165408 (2003).

[12] P. Miller and J. K. Freericks, J. Phys. Condens. Matter 13, 3187 (2001); L. Chen and J. K. Freericks, Phys. Rev. B 75, 125114 (2007); J. K. Freericks, Transport in Multilayered Nanostructures (Imperial College Press, London, 2006).

[13] M. P. Sarachik et al., Phys. Rev. B 58, 6692 (1998).

[14] R. Bulla, T. A. Costi, and D. Vollhardt, Phys. Rev. B 64, 045103 (2001).

[15] R. Bulla, T. Costi, and T. Pruschke, Rev. Mod. Phys. (to be published); arXiv:cond-mat/0701105.

[16] We use for all of our calculations (see Ref. [15]) $\Lambda=1.5$, 60 shells and 800 kept states (using particle number, $S_{z}$ and $S^{2}$ conservation).

[17] F. Gerbier, S. Foelling, A. Widera, and I. Bloch, arXiv:cond-mat/0701420v1. 